\title{
Influenza: New Insights Into an Old Disease
}

\author{
Kathleen M. N euzil, MD, M PH
}

\begin{abstract}
Address
Department of Veterans A ffairs Puget So und H ealth Care System, Medical Service 111, 1660 S. C olumbian W ay, Seattle, WA, 98108-1595, USA.

E-mail: kneuzil@ u.washington.edu
\end{abstract}

Current Infectious Disease Reports 2000, 2:224- 230

Current Science Inc. ISSN 1523-3847

Copyright $\odot 2000$ by Current Science Inc.

In the United States each year, influenza accounts for an estimated 20,000 to 40,000 deaths, nearly 300,000 hospitalizations, and millions of days lost from work. In the past few years, there has been an explosion of information in the medical literature related to influenza. This paper reviews these latest developments that enhance our understanding of influenza epidemiology and virology and expand our prevention and treatment options. Diminishing the impact of influenza in this country will require continued intense surveillance, increased use of influenza vaccine, and the availability of alternative vaccines and antivirals with the potential for broader protection against shift-and-drift strains of influenza.

\section{Introduction}

Influenza is a highly contagious disease that has the ability to cause illness in people of all ages. It has been termed "the most underrated pathogen in the devel oping world" [1]. A recent outbreak of an avian influenza virus that was highly virulent in humans has reminded us that influenza virus remains a formidable human adversary.

Advances in our knowledge of viral structure and function and host interaction have led to new insights into past influenza epidemics and to the development of new antiviral compounds and vaccines. Rigorous population-based studies and randomized controlled trials have del ineated the impact of influenza on various populations and the efficacy, effectiveness, and cost effectiveness of preventive and treatment options.

\section{Influenza Virology and Pandemic Influenza}

Three major classes of influenza virus are recognized: A, B, and $C$. Influenza $C$ is a relatively minor cause of respiratory illness, but influenza A and B strains are major causes of morbidity and mortality. Each tends to occur in well-cir- cumscribed epidemics during the winter. The severity of influenza epidemics is a function of viral dharacteristics and underlying immunity in a population. Influenza viruses are orthomyxoviruses that possess a segmented RNA genome This has al lowed periodic introduction into the human pool of viral hemagglutinins or neuraminidases from animal, usually avian, sources, leading to a novel surface glycoprotein against which large segments of the human population have no antibody. This antigenic shift in the glycoprotein structure, coupled with the ability of a virus to replicate and spread rapidly in humans, has led to pandemics of influenza, of which the Asian influenza strain of 1957 and the Hong Kong influenza strain of 1968 are the most recent examples. Variability on a yearly or biyearly basis is afforded by point mutations in the hemagglutinin structure that alter antibody recognition sufficiently to al low epidemic spread of so-called drift strains.

\section{The 1918 influenza pandemic}

Although epidemics of influenza have been recognized for centuries, the worst pandemic in recorded history occurred in 1918-1919. The 1918 influenza pandemic was remarkable for the total estimated 20 to 40 million deaths it caused, as well as its extremely high mortality rate among young, heal thy adults [2]. The devastating impact of this pandemic has al ways stimulated interest in and speculation about the influenza strain responsible for the outbreak, which occurred more than a decade before influenza virus was isolated. Serol ogic studies have long suggested that the virus was of the H1N1 type, but the first confirmation of this came from a series of studies conducted at the Armed Forces Institute of Pathology over the past several years $[3,4 \bullet \bullet]$. Using preserved autopsy material from two United States servicemen killed in the 1918 pandemic and one frozen sample obtained by in situ biopsy of the lung of a victim buried in permafrost since 1918, these researchers isolated and sequenced influenza virus RNA. The sequences were consistent with a novel H1N 1 influenzavirus A. Phylogenetic analyses suggest that the 1918 virus hemagglutinin gene is most closely related to the human and swine influenza strains of the 1930s. The authors postulate that the virus did not pass directly from an avian source to its pandemic form but rather spent some unknown amount of time adapting in a mammalian host $[3,4 \bullet \bullet, 5]$. The alternative hypothesis, that this virus may be more closely related to avian species of that time 
period, cannot be tested, because there are no known samples of avian $\mathrm{H} 1$ viruses from 1918 [6].

\section{Avian influenza}

In general, viruses with more avian characteristics, although more immunogenic in humans, are also thought to be less transmissible to humans owing to the species specificity of the influenza receptor. Thus, it had been general ly believed that viruses cross the avian-human species barrier after prior adaptation in another mammalian species, usually swine This concept was challenged in 1997, when the first documented outbreak of human respiratory disease caused by avian influenza A (H5N1) viruses occurred in Hong Kong $[7 \bullet \bullet, 8]$. In predominantly young, healthy patients, the avian influenza A H5N 1 virus caused a human influenza-like respi ratory illness with a high rate of complications. Of a total of 18 human cases, six were fatal [9]. A temporal association between exposure to live poultry and subsequent clinical H5N1 illness, and between the mass slaughter of poultry in Hong Kong and theend of new cases in humans, suggested that transmission occurred directly from birds to humans [10,11]. A subsequent cohort study, however, identified H5-antibody-positive household contacts, suggesting that human-to-human transmission may have occurred [12]. In 1999, human infection with another avian influenza, H9N2, was reported to cause mild illness in two children [13]. With 15 known hemagglutinin subtypes and nine known neuraminidases, avian species provide an extensive reservoir of influenza viruses that are novel to man. The devastation caused by the influenza pandemic of 1918 and the recent experience in Hong Kong are alarming and remind us that the threat of a future pandemic is real. Global influenzavirus surveillance in humans and animals and the development of control strategies are essential components of pandemic preparedness [14].

\section{Interpandemic Influenza}

Even in interpandemic years, the impact of influenza on society is substantial [15]. Influenza is spread primarily by respiratory droplet. During epidemics, attack rates may exceed $40 \%$ in preschool children and $30 \%$ in school-aged children. In adults, attack rates average $10 \%$ to $20 \%$. Children play an important role in the spread of influenza, with school-aged children being the main introducers of influenza into households. The arrival of influenza in a community can be appreciated by increased school absenteeism and an influx of people with febrile respiratory illness with a major systemic component into healthcare facilities. Each year in this country, influenza accounts for excess outpatient visits, excess antibiotic prescriptions, and millions of days lost from school and work. Most important, epidemics are reflected by excess morbidity and mortality. During influenza season, there are approximatel y 300,000 excess hospitalizations and between 20,000 and 40,000 excess deaths, with mortality occurring primarily in the elderly [15-17].
Although mortality statistics are sensitive indicators of the impact of influenza, they underestimate the overall heal th impact of influenza on other populations [17]. A series of recent articles more accurately define the serious consequences of influenza in several populations, including children and pregnant women $[18 \cdot, 19 \cdot, 20]$.

\section{Influenza morbidity in children}

Acute respiratory disease is the most common reason for outpatient visits and hospital izations among children in the United States. Although respiratory syncytial virus is recognized as the most frequent cause of lower respiratory tract disease leading to hospitalization in infants and young children, two recent retrospective cohort studies demonstrate that influenza can al so be a serious disease in this age group $[18 \bullet, 19 \bullet]$. In these two studies of healthy children with differing socioeconomic backgrounds (one group enrolled in either one of two West Coast heal th maintenance organizations and one group enrolled in Tennessee Medicaid), the authors showed that children aged younger than 2 years had hospital ization rates attributable to influenza that were comparable to rates in high-risk adults $[18 \bullet, 19 \bullet]$. Furthermore, in the Tennessee Medicaid study, for every 100 children aged younger than 15 years, there was an average of six to 15 outpatient visits and three to nine antibiotic courses attributable to influenza annual ly. Influenza accounted for $10 \%$ to $30 \%$ of the winter excess of antibiotic prescriptions in this population. These authors conclude that young children suffer substantial morbidity from influenza, and improved influenza prevention in children is needed.

\section{Influenza morbidity in pregnant women}

Although influenza-associated excess mortal ity among pregnant women was documented during the 1918-1919 and 1957-1958 pandemics, it has not been documented during interpandemic periods. Case reports and papers on small case series published since that time have described women in the third trimester and early puerperium who died from influenza-related complications, possibly related to the physiologic or immunologic changes that accompany pregnancy. A recent large, retrospective cohort study of all women aged 15 to 44 years enrolled in Tennessee Medicaid during 17 interpandemic influenza seasons reported that otherwiselow-risk women in the first trimester of pregnancy or first 6 months postpartum had a risk of influenzarelated hospitalizations similar to that of nonpregnant women. Otherwise low-risk women in the second or third trimester of pregnancy experienced approximately 14 and 23 excess acute cardiopulmonary hospital izations, respectively, per 10,000 women in an average influenza season, however-a rate comparable to that seen in other high-risk groups for whom vaccine is recommended [20]. There were no deaths from acute cardiopul monary causes during influenza season among pregnant women in this study. Using data from this study, the researchers estimated than an average of one to 
two hospitalizations could be prevented for every 1000 pregnant women vaccinated, and pregnant women are now among the high-risk groups targeted for influenza immunization (Table 1).

\section{Influenza Vaccine}

Inactivated influenza vaccines are prepared that reflect currently circulating strains of influenza $A$ and B. Vacanation in young adults is $70 \%$ to $90 \%$ efficacious in preventing clinical illness caused by the sameor a closely related influenza virus. Although this efficacy is considerably diminished in the el derly, vaccination reduces the number of hospitalizations and mortality in this population and is cost effective [21]. Available vaccines are highly purified, and in multiple randomized, double-blind trials, the only significant side effect associated with vaccination was arm soreness. Egg allergy is the only contraindication to vaccination. Because influenza vaccine contains only noninfectious viruses, it cannot cause influenza. Although the 1976 swine influenza vaccine was associated with an increased frequency of Guillain-Barré syndrome, recent influenza vaccination programs (for the seasons of 1992-1993 and 1993-1994) were associated with only one or two additional cases of the Guillain-Barré syndrome per 1 million vaccinated persons $[22,23]$.

\section{Recommendations for use}

The approach to influenza control in this country has been the administration of inactivated influenza vaccines to persons who are at high risk for influenza-related complications, or to those persons who can transmit the disease to persons at high risk [24••] (Table 1). Although almost $70 \%$ of people older than the age of 65 years in the United States have recei ved the influenza vaccine in recent years, only an estimated $30 \%$ to $40 \%$ of younger high-risk persons and healthcare workers receive the vaccine [25••]. Clearly, more effective strategies for insuring better influenza vaccine coverage of these latter groups are needed.

\section{Influenza vaccine and HIV infection}

Although limited information exists regarding the incidence of influenza and vaccine efficacy in HIV-infected persons, recent evidence suggests that the benefits of vaccination outweigh the risk. Early reports of detrimental effects of influenza immunization on viral load measurements have not proven to be clinically relevant. Several case reports suggest that influenza symptoms are prolonged and the risk for complications from influenza increased for some HIV-infected persons. A recent retrospective study of young and middleaged women enrolled in the Tennessee Medicaid program found that women with HIV infection had a higher risk of influenza-attributable hospitalizations than women with chronic heart or lung diseases; the estimates were as high as 30 hospital izations for every 1000 HIV-infected women [16]. In a recent

\section{Table 1. Target groups for vaccination}

Persons at high risk for influenzavirus-related complications Persons aged 50 years or older

Residents of nursing homes and other chronic-care facilities housing persons of any age with chronic medical conditions

Adults and children with chronic disorders of the pulmonary or cardiovascular systems, including asthma

Adults and children who have required regular medical follow-up or hospitalization during the preceding year owing to chronic metabolic diseases (including diabetes mellitus), renal dysfunction, hemoglobinopathies, or immunosuppression

Children and teenagers (aged 6 months to 18 years) who are receiving long-term aspirin therapy and may be at risk for developing Reye's syndrome after influenza

W omen who will be in the second or third trimester of pregnancy during the influenza season

Persons able to transmit influenzavirus to those at high risk

Physicians, nurses, and other personnel in both hospital and outpatient-care settings

Employees of nursing homes and chronic-care facilities having contact with patients or residents

Employees of assisted living and other residences for persons in high-risk groups

Persons who provide home care to persons in high-risk groups

Household members (including children) of persons in high-risk groups

$O$ ther groups to consider

Persons infected with HIV

Breastfeeding mothers

Travelers

General population

*Recommendations of the A dvisory C ommittee on Immunization Practices for the U se of Influenza Vaccine.

randomized, double-blind, placebo-controlled trial of pre dominantly young men with HIV infection, those who recei ved influenza vaccine had modest serologic responses. The vaccinewas highly dinically effective, however, with 10 placebo recipients and no vaccine recipients having symptomatic laboratory-confirmed influenza A [26].

\section{Limitations}

Although achieving control of influenza through vaccination is a laudable goal, it is problematic. As previously discussed, the influenza virus is capable of antigenic change, requiring that a new vaccine be developed yearly that best matches that year's predicted influenza strain. Likewise, it is impossible to predict before any given influenza season whether it will be a "mild" or "severe" year (Fig. 1). For these reasons, comprehensive influenza vaccine campaigns must occur each year. Patients, their physicians, and insurance providers must understand that one must look at the clinical and cost effectiveness of influenza vaccination over a range of years, never over a single year. This variability is one reason for the often-contradictory findings in cost- 


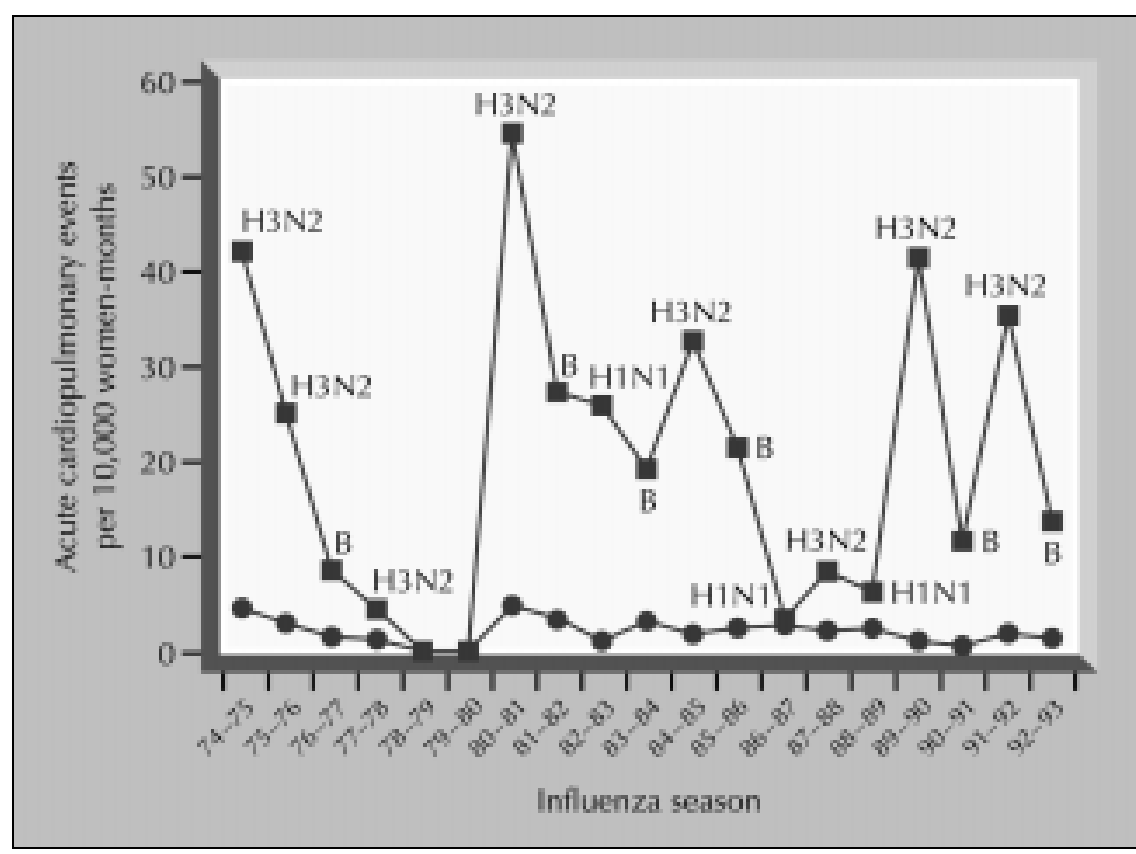

Figure 1. Excess acute cardiopulmonary events (hospitalizations and deaths) during influenza season by year in low- $(\bullet)$ and high(घ) risk women aged 15 to 64 years enrolled in the Tennessee Medicaid Program 1974 to 1993. This graph demonstrates the variable impact of influenza in this cohort from year to year. Strain of virus is shown for each season. (Adapted from Neuzil et al. [16].)

effectiveness studies of the use of influenza vaccine in heal thy adults and healthcare workers [27-29]. Clearly, the benefits of influenza vaccine will be greatest in a season marked by high attack rates and a good vaccine match. Additional ly, we must recognize that the vacanation policy in this country is designed to protect individuals from the complications of influenza. If the goal is to interrupt transmission, then vaccination must be directed toward those members of the community, predominantly school-aged children, who are integral to the dissemination of the virus.

\section{Live and attenuated intranasal vaccines}

Several ways to overcome the aforementioned limitations of our current method of influenza control include better methods of predicting future circulating influenza strains [30], the use and development of vaccines that will provide broader protection against drift-and-shift strains, and administering vaccineto a larger segment of the population.

Intranasal ly admi nistered, cold-adapted, live attenuated vaccines have the potential advantage of inducing a broad mucosal and systemic immune response [31]. Their ease of administration and the acceptability of an intranasal route of administration may serve to increase the total number of influenza vaccine recipients. In a recent study of children aged 15 to 71 months, intranasal vaccine was $93 \%$ effective in preventing culture-confirmed influenza. The vaccinated children had significantly fewer febrile episodes, including $30 \%$ fewer episodes of febrile otitis media [32 • ]. In a study of heal thy adults younger than 65 years of age, Nichol et al. [33] showed that intranasally administered live attenuated vaccines significantly reduced the numbers of severe febrile illnesses and febrile upper respiratory tract illnesses, and that recipients of these vaccines missed fewer days of work and had fewer healthcare pro- vider visits than placebo recipients. These results are no more impressive than those seen with inactivated vaccine in healthy adults. In a 5-year study, it was found that the trival ent inactivated vaccine and bivalent live attenuated vaccine were approximately equival ent in terms of effectiveness [34]. During the period of the Nichol study, however, the circulating virus demonstrated significant drift from the vaccine virus, suggesting the possibility of broader immunity [33]. These vaccines may be licensed by the 2001-2002 winter season.

\section{Antiviral Agents}

Perhaps the most publicized of the many advances related to influenza in the past year has been the development and Food and Drug Administration approval of the first new dass of antiviral drugs in 30 years, the neuraminidase inhibitors. The neuraminidase inhibitors are the result of computerassisted drug design based on the crystal structure of the neuramini dase target protein [35]. Inhibition of neuraminidase enzymatic action causes virus particles to aggregate at thecell surface and with each other. Theenzyme-activesite is highly conserved across influenza $A$ and $B$ viruses.

Before 1999, amantadine and rimantadine, with efficacy agai nst influenza $A$ but not influenza B, were the only drugs available for the prevention and treatment of influenza and were not widely used. These agents are $70 \%$ to $90 \%$ effective in preventing disease in adults and children when admi nistered prophylactically throughout the influenza season. Treatment with amantadine and rimantadine shortens the duration of clinical influenza illness by approximately 1 day [36]. The disadvantages of these agents include the rapid development of viral resistance, inactivity agai nst influenza $B$, and neurologic side effects. 


\begin{tabular}{|c|c|c|c|c|c|}
\hline Drug & $\begin{array}{l}\text { Mechanism of } \\
\text { action }\end{array}$ & Major side effects & FDA indication & Dose & Dose reductions \\
\hline Amantadine & $\begin{array}{l}\text { Interferes with } \\
\text { M2 protein } \\
\text { (influenza A only) }\end{array}$ & $\begin{array}{l}\text { >10\% C N S-related } \\
\text { (nervousness, anxiety, } \\
\text { difficulty } \\
\text { concentrating) }\end{array}$ & $\begin{array}{l}\text { Prevention and } \\
\text { treatment }\end{array}$ & $\begin{array}{l}100 \text { mg twice } \\
\text { daily, oral }\end{array}$ & $\begin{array}{l}\text { Elderly persons, } \\
\text { those with renal } \\
\text { impairment* }\end{array}$ \\
\hline Rimantadine & $\begin{array}{l}\text { Interferes with } \\
\text { M2 protein } \\
\text { (influenza A only) }\end{array}$ & $\begin{array}{r}<10 \% \text { C N S-related } \\
\text { (similar to above) }\end{array}$ & $\begin{array}{l}\text { Prevention and } \\
\text { treatment }\end{array}$ & $\begin{array}{l}100 \text { mg twice } \\
\text { daily, oral }\end{array}$ & $\begin{array}{l}\text { Elderly persons, } \\
\text { those with } \\
\text { severe renal } \\
\text { impairment }^{\dagger}\end{array}$ \\
\hline Zanamivir & $\begin{array}{l}\text { Inhibits } \\
\text { neuraminidase } \\
\text { (influenza } A \text { and } B \text { ) }\end{array}$ & $\begin{array}{l}\text { Bronchospasm in } \\
\text { patients with asthma } \\
\text { (unknown incidence) }\end{array}$ & Treatment & $\begin{array}{l}10 \text { mg twice daily } \\
\text { for } 5 \text { days, inhaled }\end{array}$ & \\
\hline O seltamivir & $\begin{array}{l}\text { Inhibits } \\
\text { neuraminidase } \\
\text { (influenza } A \text { and } B \text { ) }\end{array}$ & $\begin{array}{l}>10 \% \text { gastrointestinal } \\
\text { (nausea, vomiting) }\end{array}$ & Treatment & $\begin{array}{l}75 \text { mg twice daily } \\
\text { for } 5 \text { days, inhaled }\end{array}$ & $\begin{array}{l}\text { Those with renal } \\
\text { impairment }^{\ddagger}\end{array}$ \\
\hline \multicolumn{6}{|c|}{$\begin{array}{l}{ }^{*} \text { C reatinine clearance } \leq 50 \mathrm{~mL} / \mathrm{min} / 1.73 \mathrm{~m}^{2} \text {. } \\
{ }^{\dagger} \text { C reatinine clearance } \leq 10 \mathrm{~mL} / \mathrm{min} / 1.73 \mathrm{~m}^{2} . \\
{ }^{\ddagger} \mathrm{C} \text { reatinine clearance } \leq 30 \mathrm{~mL} / \mathrm{min} / 1.73 \mathrm{~m}^{2} \text {. } \\
\text { CN S - central nervous system; FD A - Fooc }\end{array}$} \\
\hline
\end{tabular}

\section{Neuraminidase inhibitors}

Zanamivir and oseltami vir are the neurami nidase inhibitors recently licensed for the treatment of influenza $A$ and $B$ infection. Although there are no studies comparing the two neuraminidase inhibitors with amantadine or rimantadine, theoretical advantages of neuraminidase inhibitors over the latter agents include efficacy against type B viruses, rare development of resistance, and a different side effect profile

Three large, randomized, placebo-controlled trials examining the efficacy and safety of zanamivir in the treatment of influenza virus infections have been published to date $[37,38 \bullet, 39]$. Participants were generally young (mean ages 30 to 38 years) and heal thy. Zanami vir reduced the severity and duration of influenza illness by a day or more when given within 48 hours of onset of symptoms. More substantial reductions in clinical illness were seen in those patients who received therapy within 30 hours of onset of symptoms, in those patients who presented with febrile illness, and in the small subset of patients with underlying high-risk conditions, predominantly respiratory disease In this high-risk subset, zanamivir significantly reduced pulmonary complications from $46 \%$ to $14 \%$ and associated antibiotic use from $38 \%$ to $14 \%$ [38•]. Viral titers of nasal washings in the groups given inhaled zanamivir were significantly lower than those in the placebo group, suggesting the possibility for reduced virus transmission [37].

In a randomized, placebo-controlled trial of treatment of experimental influenza in young, healthy adults, oral oseltamivir reduced viral shedding and significantly reduced illness burden and biochemical markers of inflammation. As compared with placebo recipients, fewer osel tamivir recipients used acetaminophen [40•]. As-yet unpublished studies using oseltamivir for the treatment of naturally acquired influenza corroborate these results.

It is difficult to know how the clinical efficacy of these agents will translate into community effectiveness. Rapid, site-based diagnostic tests are not widely available, and therefore these drugs will need to be given rapidly after symptom onset, based primarily on clinical criteria. In the clinical trials, $60 \%$ to $70 \%$ of patients had laboratory-confirmed influenza, suggesting that in clinical practice, many people with diseases other than influenza will be exposed to these agents. The cumulative experience with these drugs remains limited; thus, postmarketing surveillancefor less common side effects is essential. Finally, no studies are available comparing the two neuraminidase inhibitors with one another or with amantadine and rimantadine. The choice of which drug to use in any given patient will likely be based on side-effect profile, cost, and ease of administration (Table 2).

The most important role for neuraminidase inhibitors may be as an adjunct to immunization in preventing influenza during years when the vaccine strain is a poor match for the circulating influenza strain or during nursing home or institutional outbreaks. Although both agents have shown efficacy in this regard, neither drug has yet been approved by the Food and Drug Administration for this indication [41-43].

\section{Conclusions}

Influenza remains a major cause of morbidity and mortality across a broad segment of the population. The mutability and zoonotic reservoir of the virus challenge our ability to eradicate this infection. Although inactivated influenza 
vaccine should remain the cornerstone of influenza control programs, new vaccines and antivirals offer alternative methods of prevention with the potential for broader protection against shift-and-drift strains of influenza virus.

References and Recommended Reading Papers of particular interest, published recently, have been highlighted as:

- Of importance

-. Of major importance

1. Bartlett JG: Update in infectious diseases. Ann Intern M ed 1999, 131:273-280.

2. Glezen WP: Emerging infections: pandemic influenza. Epidemiol Rev 1996, 18:64-76.

3. Taubenberger JK, Reid AH, Krafft AE, et al.: Initial genetic characterization of the 1918 "Spanish" influenza virus. Science 1997, 275:1793-1796.

4. $\bullet$ Reid AH, Ganning TG, Hultin JV, Taubenberger JK: Origin and evolution of the 1918 "Spanish" influenza virus hemagglutinin gene. Proc N atl Acad Sci U S A 1999, 96:1651-1656.

This paper reports the complete sequence of the hemaggl utinin gene of the 1918 influenza virus. Although unarguably historically important, the paper is al so significant for the molecular biology techniques used on preserved tissue, which have implications beyond the field of influenza research.

5. Reid $\mathrm{AH}$, Taubenberger JK: The $\mathbf{1 9 1 8}$ flu and other influenza pandemics: "over there" and back again. Lab Invest 1999, 79:95-101.

6. Webster RG: 1918 Spanish influenza; the secrets remain elusive. Proc N atl Acad Sci U S A 1991, 96:1164-1166.

7. • Subbarao K, Klimov A, Katz J, et al.: Characterization of an avian influenza $A$ (H5N1) virus isolated from a child with a fatal respiratory illness. Science 1998, 279:393-396.

This paper is a grim reminder that influenza is a zoonosis, and the potential for future avian influenza outbreaks is real.

8. Claas ECJ, Osterhaus $A D$, van Beek $R$, et al.: Human influenza A H 5N1 virus related to a highly pathogenic avian influenza virus. Lancet 1998, 351:472-477.

9. Yuen KY, Chan PKS, Peiris M, et al.: Clinical features and rapid viral diagnosis of human disease associated with avian influenza A H5N 1 virus. Lancet 1998, 351:467-471.

10. Mounts AW, Kwong H, Izurieta HS, et al.: Case- control study of risk factors for avian influenza A (H5N1) disease, Hong Kong, 1997. J Infect D is 1999, 180:505-508.

11. Belshe RB: Influenza as a zoonosis: how likely is a pandemic? Lancet 1998, 351:460-461.

12. Katz JM, Lim W, Bridges CB, et al.: Antibody response in individuals infected with avian influenza $A$ (H5N1) viruses and detection of anti- $\mathrm{H} 5$ antibody among household and social contacts. J Infect D is 1999, 180:1763-1770.

13. Peiris $M$, Yuen $K Y$, Leung $C W$, et al.: Human infection with influenza H 9N2. Lancet 1999, 354:916-917.

14. Snacken R, Kendal AP, Haaheim LR, Wood JM: The next influenza pandemic: lessons from Hong Kong, 1997. Emerg Infect Dis 1999, 5:195-203.

15. Simonsen L, Clarke MJ, Schonberger LB, et al.: Pandemic versus epidemic influenza mortality: a pattern of changing age distribution. J Infect D is 1998, 178:53-60.

16. Neuzil KM, Reed GW, Mitchel EF, Griffin MR: Influenzaassociated morbidity and mortality in young and middleaged women. JAM A 1999, 281:901-907.

17. Perrotta DM, Decker M, Glezen WP: Acute respiratory disease hospitalizations as a measure of impact of epidemic influenza. Am J Epidemiol 1985, 122:468-476.
18. Izurieta HS, Thompson WW, Kramarz P, et al.: Influenza and the rates of hospitalizations for respiratory disease among infants and young children. N Engl J M ed 2000, 342:323-329. This paper, together with thefollowing, details the substantial impact of influenza on young, healthy children, a group for whom yearly influenza vaccination has not been a priority.

19. - Neuzil KM, Mellen BG, Wright PF, et al.: The effect of influenza on hospitalizations, outpatient visits, and courses of antibiotics in children. N Engl J M ed 2000, 342:225-231.

This paper, together with the preceding reference, details the substantial impact of influenza on young, healthy children, a group for whom yearly influenza vaccination has not been a priority.

20. Neuzil KM, Reed GW, Mitchel EF, et al.: Impact of influenza on acute cardiopulmonary hospitalizations in pregnant women. Am J Epidemiol 1998, 148:1094-1102.

21. Nichol KL, Margolis, KL, Wuorenma J, Von Sternberg T: The efficacy and cost-effectiveness of vaccination against influenza among elderly persons living in the community. N Engl J M ed 1994, 331:778-784.

22. Lasky T, Terracciano GJ, Magder L, et al.: The Guillain-Barre syndrome and the 1992-1993 and 1993-1994 influenza vaccines. N Engl J M ed 1998, 339:1797-1802.

23. Ropper $A H$, Victor $M$ : Influenza vaccination and the GuillainBarre syndrome. N Engl J M ed 1998, 339:1845-1846.

24. $\bullet$ Centers for Disease Control and Prevention: Prevention and control of influenza. M M W R M orb M ortal W kly Rep 1999, 48:1-28.

A comprehensive, annually updated, and well-referenced review of influenza vaccination recommendations by the Advisory Committee for Immunization Practices of the Centers for Disease Control and Prevention.

25. Marwick C: New recommendation for adult immunization [editorial]. JAM A 1999, 282:2199.

This short editorial explains the decision to change the age for universal immunization of adults to 50 years and older. This represents a major step toward broadening our use of influenza vaccine

26. Tasker SA, Treanor JJ, Paxton WB, Wallace MR: Efficacy of influenza vaccination in HIV-infected persons. Ann Intern M ed 1999, 131:430-433.

27. Nichol KL, Lind A, Margolis KL, et al.: The effectiveness of vaccination against influenza in healthy, working adults. N Engl J M ed 1995, 333:889-893.

28. Weingarten $\mathrm{S}$, Staniloff $\mathrm{H}$, Ault $\mathrm{M}$, et al.: Do hospital employees benefit from the influenza vaccine? J Gen Intern M ed 1988, 3:32-37.

29. Saxen H, Virtanen M: Randomized, placebo-controlled double-blind study on the efficacy of influenza immunization on absenteeism of health care workers. Pediatr Infect Dis J 1999, 18:779-783.

30. Bush RM, Bender CA, Subbarao K, et al.: Predicting the evolution of human influenza A. Science 1991, 286:1921-1925.

31. Boyce TG, Gruber WC, Coleman-Dockery SD, et al.: Mucosal immune response to trivalent live attenuated intranasal influenza vaccine in children. Vaccine 1999, 18:82-88.

32. • Belshe RB, Mendelman PM, Treanor J, et al.: The efficacy of live attenuated, cold-adapted, trivalent, intranasal influenzavirus vaccine in children. $N$ Engl J M ed 1998, 338:1405-1412.

This randomized, placebo-controlled trial demonstrated the dinical effectiveness of liveattenuated vaccines in children. Licensure of these vaccines is anticipated soon.

33. Nichol KL, Mendelman PM, Mallon KP, et al.: Effectiveness of live, attenuated intranasal influenza virus vaccine in healthy, working adults. JAM A 1999, 282:137-144.

34. Edwards KM, Dupont WD, Westrich MK, et al.: A randomized controlled trial of cold-adapted and inactivated vaccines for the prevention of influenza A disease. J Infect D is 1994, 169:68-76.

35. Von Itzstein M, Wu WY, Kok GB, et al.: Rational design of potent sialidase-based inhibitors of influenza virus replication. $N$ ature 1993, 363:418-423. 
36. Dolin R, Reichman RC, Madore HP, et al.: A controlled trial of amantadine and rimantadine in the prophylaxis of influenza A infection. N Engl J M ed 1982, 307:580-584.

37. Hayden FG, Osterhaus AD, Treanor JJ, et al.: Efficacy and safety of the neuraminidase inhibitor zanamivir in the treatment of influenzavirus infections. N Engl J M ed 1997, 337:874-880.

38. The MIST Study Group: Randomized trial of efficacy and safety of inhaled zanamivir in treatment of influenza A and B virus infections. Lancet 1998, 352:1877-1881.

This is the first published study to demonstrate that an antiviral for influenza can reduce complications of influenza and decrease antibiotic use in a high-risk subset of patients.

39. Monto AS, Fleming DM, Henry D, et al.: Efficacy and safety of the neuraminidase inhibitor zanamivir in the treatment of influenza A and B virus infections. J Infect D is 1999, 180:254-261.
40. - Hayden FG, Treanor JJ, Fritz RS, et al.: Use of the oral neuraminidase inhibitor oseltamivir in experimental human influenza. JAM A 1999, 282:1240-1246.

This meticul ous challenge study details the efficacy of oseltamivir in the prevention and treatment of influenza, as well as its effect on viral shedding and host cytokine response

41. Hayden FG, Atmar RL, Schilling M, et al.: Use of the selective oral neuraminidase inhibitor oseltamivir to prevent influenza. N Engl J M ed 1999, 341:1336-1343.

42. Monto AS, Robinson DP, Herlocher ML, et al.: Zanamivir in the prevention of influenza among healthy adults. JAM A 1999, 282:31-35.

43. Schilling M, Povinelli L, Krause P, et al.: Efficacy of zanamivir for chemoprophylaxis of nursing home influenza outbreaks. Vaccine 1998, 16:1771-1774. 